\section{Wissenswertes für Allergologen}

D ie Allergologie ist seit vielen Jahren integraler Bestandteil unseres Fachgebietes. Sie stellt durch die Zunahme der Erkrankungen und die Fülle neuer wissenschaftlicher Erkenntnisse zu Ätiologie, Diagnostik und Therapie eine der großen Herausforderungen auch innerhalb der Hals-Nasen-Ohrenheilkunde dar. Gerade durch die Interdisziplinarität werden typische Krankheitsbilder aus unserem Fachgebiet häufig fachfremd und damit nicht wirklich fachärztlich im ursprünglichen Sinne des Wortes behandelt. Entsprechend wichtig ist es für jeden HNO-
Arzt, aktualisiertes Wissen aus den verschiedenen Gebieten der Allergologie „zur Hand“ zu haben, um den laienwissenschaftlich und über Internet vor"gebildeten" Patienten seriös zu beraten.

Zur Allergologie existiert eine Fülle an Literatur, die die unterschiedlichen Interessensgruppen bedient; in diesem Kontext ist das Allergologie-Handbuch, wie der Name schon andeutet, auch in der zweiten Auflage als Nachschlagewerk $\mathrm{zu}$ sehen, in dem alles Wesentliche zu Grundlagen, Epidemiologie, Diagnostik, zu Krankheitsbildern selbst und zur The-

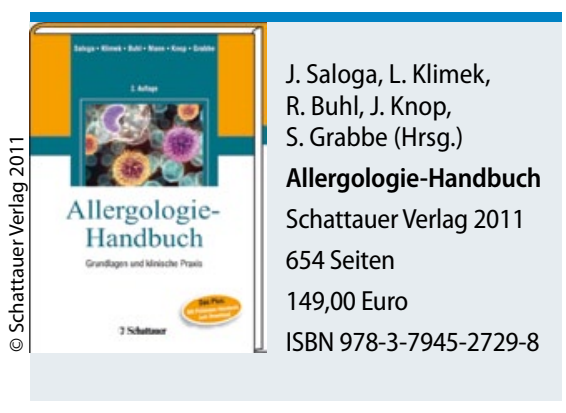

rapie von namhaften deutschen Allergologen zusammengefasst wurde. In diesem Sinne sollte es zum Nachschlagen in der Bibliothek des allergologisch tätigen Arztes vorliegen.

Prof. Dr. med. Gerhard Grevers

\title{
Überarbeitetes Standardwerk zu Stimmstörungen
}

D ieses Lehrbuch gibt dem interessierten Facharzt einen umfassenden Überblick über alle Stimmformen und Stimmstörungen in jedem Lebensalter sowie über die dazu passenden diagnostischen Methoden.

Für das von Günter Wirth begründete Standardwerk konnten namhafte Co-Autoren wie Tadeus Nawka und Lutz-Christian Anders gewonnen werden. Damit ist die vorliegende fünfte Auflage nicht nur eine erweiterte, sondern vollkommen überarbeitete und komplettierte Auflage. Dies betrifft besonders die funktionellen und phonochirurgischen Behandlungsmethoden.

Das Buch „Stimmstörungen“" sollte jeder benutzen, der sich mit Stimme beschäftigt. Dies betrifft nicht nur Ärzte und Logopäden, sondern zielt auch auf Sprachheilpädagogen und Sprechwissenschaftler.

Der Text ist sehr gut verständlich. Die endoskopischen Abbildungen wären al-

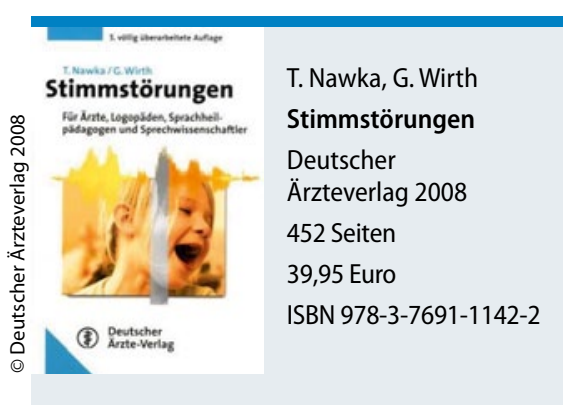

lerdings in Farbe übersichtlicher. Dafür ist der Preis von 39,95 € angemessen.

Prof. Dr. med. Karin Schorn

\section{Husten, Schnupfen, Fieber - was Ihre Patienten nun wissen wollen}

Wenn das Wetter Kapriolen schlägt, macht so manches Immunsystem schlapp. Dann drängen sich in Ihrer Praxis wieder Patienten, die wissen wollen, wie sie sich besser schützen und ihre Beschwerden lindern können. In einer Umfrage haben Sie uns die häufigsten Fragen genannt, die Ihre Patienten in diesem Zusamenhang an Sie stellen. Daraus ist nun eine Broschüre entstanden, die diese Fragen beantwortet.

D ie Patientenbroschüre „20 Fragen 20 Antworten“ zum Thema „Erkältung und fieberhafter Infekt“ erklärt dabei nicht nur, ab wann eine erhöhte Körpertemperatur wirklich als Fieber bezeichnet wird und bei welchen Komplikationen besser der Arzt zu Rate gezogen werden sollte. Auch die Frage „Was unterscheidet einen grippalen Infekt von einer richtigen
Grippe?" wird in der 28-seitigen Broschüre beantwortet.

Durch die Broschüre sollen die wichtigsten Probleme ausführlich dargestellt und beantwortet werden, sodass in der Sprechstunde mehr Zeit für gezieltere Fragen bleibt. Daneben wird die Arztempfehlung zur Selbstmedikation durch das Grüne Rezept unterstützt.

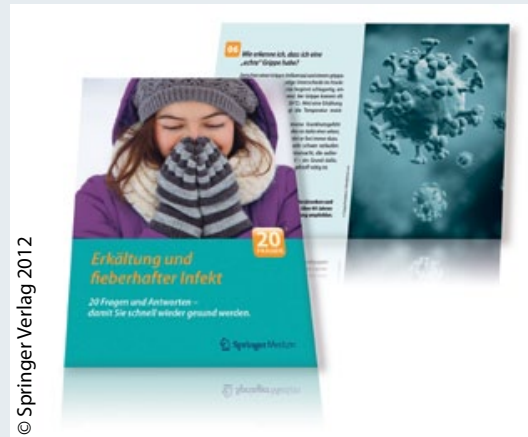

Die Broschüre kann von allen Ärzten (immer im Paket von 100 Exemplaren) fürs Wartezimmer bestellt werden. Per E-Mail an: vertrieb@springer.com oder per Fax an: 06102 / 5062 40, Stichwort „Erkältung und fieberhafter Infekt“. 